ANNALES

POLONICI MATHEMATICI

$84.1(2004)$

\title{
A uniqueness result for an inverse problem
}

\author{
by Robert Dalmasso (Grenoble)
}

\begin{abstract}
We establish a uniqueness result for an overdetermined boundary value problem. We also raise a new question.
\end{abstract}

1. Introduction. Let $\Omega \subset \mathbb{R}^{2}$ be a smooth bounded simply-connected open set. We consider the following elliptic boundary value problem:

$$
\begin{aligned}
\Delta u & =-\lambda u-\mu & & \text { in } \Omega \\
u & =0 & & \text { on } \partial \Omega,
\end{aligned}
$$

where $\lambda$ and $\mu$ are real constants. An interesting problem is to examine whether one can identify the constants $\lambda$ and $\mu$ from knowledge of the normal flux $\partial u / \partial n$ on $\partial \Omega$ corresponding to some nontrivial solution of (1)-(2). For more general right hand sides this inverse problem arises for instance in plasma physics in connection with the modelling of Tokamaks [1]. But even in the very particular case of an affine term the problem is difficult. It is well known that if $\Omega$ is a disk then such identification of $(\lambda, \mu)$ is completely impossible, even in the case where a sign is imposed on the right hand side of the equation: It is shown in [4] that there is a continuum of coefficient pairs $\left(\lambda, \mu_{\lambda}\right) \in \mathbb{R}^{2}$, and therefore a continuum of affine functions, which give rise to the same normal derivative on the boundary. We refer the reader to paper [4] for a more detailed description of the problem in general and the difficulties encountered.

A partial answer to this problem was first obtained by Vogelius in [4], and more recently we have also given a contribution [2], [3]: Under some conditions on the domain and on the normal derivative, there exist at most finitely many pairs of coefficients.

In order to formulate and explain our goal we first describe the construction of the continuum of coefficient pairs $\left(\lambda, \mu_{\lambda}\right) \in \mathbb{R}^{2}$ in the case where $\Omega$ is the unit disk. Let $J_{z}$ denote the $z$ th Bessel function. For any $\lambda>0$ such

2000 Mathematics Subject Classification: 35J05, 35R30.

Key words and phrases: inverse problem, Schiffer conjecture. 
that $\sqrt{\lambda}$ is not a zero of $J_{1}$ we define the function

$$
u_{\lambda}(x)=\frac{J_{0}(\sqrt{\lambda}|x|)-J_{0}(\sqrt{\lambda})}{2 \sqrt{\lambda} J_{1}(\sqrt{\lambda})}, \quad|x|<1 .
$$

For $\lambda<0$ we define the function

$$
u_{\lambda}(x)=-i \frac{J_{0}(i \sqrt{-\lambda}|x|)-J_{0}(i \sqrt{-\lambda})}{2 \sqrt{-\lambda} J_{1}(i \sqrt{-\lambda})}, \quad|x|<1 .
$$

We recall that $J_{1}$ has only real zeros [5, pp. 482-483]. We easily verify that $u_{\lambda}$ is a solution of problem (1)-(2) such that

$$
\frac{\partial u_{\lambda}}{\partial n}=-\frac{1}{2} \quad \text { on } \partial \Omega
$$

with the constant $\mu=\mu_{\lambda}$ given by

$$
\mu_{\lambda}= \begin{cases}\frac{\sqrt{\lambda} J_{0}(\sqrt{\lambda})}{2 J_{1}(\sqrt{\lambda})}, & \lambda>0, \\ \frac{i \sqrt{-\lambda} J_{0}(i \sqrt{-\lambda})}{2 J_{1}(i \sqrt{-\lambda})}, & \lambda<0 .\end{cases}
$$

The function (3) (resp. (4)) has a removable singularity at $\lambda=0$, and the corresponding solution is

$$
u_{0}(x)=\frac{1}{4}\left(1-|x|^{2}\right), \quad|x|<1 .
$$

Now we notice that $u_{0}$ is the torsion function relative to the unit disk. We state the following problem.

PROBLEM. Let $\chi$ be the torsion function relative to $\Omega$, that is,

$$
\begin{aligned}
\Delta \chi+1=0 & \text { in } \Omega, \\
\chi=0 & \text { on } \partial \Omega .
\end{aligned}
$$

What can we say about the existence of $(\lambda, \mu) \in \mathbb{R}^{2}$ and $u$ satisfying $(1)-(2)$ and

$$
\frac{\partial u}{\partial n}=\frac{\partial \chi}{\partial n} \quad \text { on } \partial \Omega ?
$$

The construction above shows that, when $\Omega$ is the unit disk, there is a continuum of coefficient pairs $\left(\lambda, \mu_{\lambda}\right)$ and $u_{\lambda}$ which solve the problem. Using only elementary facts we shall prove the following result.

THEOREM 1. Assume that $\Omega$ is a true ellipse. If there exist $\lambda, \mu \in \mathbb{R}$ and $u$ satisfying (1)-(2) and (5), then $\lambda=0, \mu=1$ and $u=\chi$. 
2. Proof of Theorem 1. We have

$$
\Omega=\left\{x=\left(x_{1}, x_{2}\right) \in \mathbb{R}^{2}: \frac{x_{1}^{2}}{a^{2}}+\frac{x_{2}^{2}}{b^{2}}<1\right\}
$$

$(a>b>0)$ and

$$
\chi(x)=\frac{a^{2} b^{2}}{2\left(a^{2}+b^{2}\right)}\left(1-\frac{x_{1}^{2}}{a^{2}}-\frac{x_{2}^{2}}{b^{2}}\right) .
$$

Let

$$
x_{1}(\theta)=a \cos \theta, \quad x_{2}(\theta)=b \sin \theta, \quad \theta \in[0,2 \pi],
$$

be a parametrization of $\partial \Omega$. The curvature of $\partial \Omega$ at $x(\theta)=\left(x_{1}(\theta), x_{2}(\theta)\right)$ is given by

$$
\kappa(\theta)=\frac{a b}{\left(a^{2} \sin ^{2} \theta+b^{2} \cos ^{2} \theta\right)^{3 / 2}},
$$

and the exterior normal $n=\left(n_{1}, n_{2}\right)$ is given by

$$
n_{1}=\frac{b \cos \theta}{\left(a^{2} \sin ^{2} \theta+b^{2} \cos ^{2} \theta\right)^{1 / 2}}, \quad n_{2}=\frac{a \sin \theta}{\left(a^{2} \sin ^{2} \theta+b^{2} \cos ^{2} \theta\right)^{1 / 2}} .
$$

We define

$$
\phi(\theta)=\frac{\partial \chi}{\partial n}(x(\theta))=-\frac{a b}{a^{2}+b^{2}}\left(a^{2} \sin ^{2} \theta+b^{2} \cos ^{2} \theta\right)^{1 / 2},
$$

and

$$
d s=\left(a^{2} \sin ^{2} \theta+b^{2} \cos ^{2} \theta\right)^{1 / 2} d \theta .
$$

Now let $u$ be a solution of (1)-(2) and (5). We shall use a formula established in [2, Lemma 2.42 ), p. 782] (see also [3]).

Lemma 1. We have

$\lambda \int_{0}^{2 \pi} \phi^{2}\left(x_{1} n_{2}-x_{2} n_{1}\right) n_{1} n_{2} d s+\mu^{2} \int_{0}^{2 \pi}\left(x_{1} n_{2}-x_{2} n_{1}\right) n_{1} n_{2} d s+a_{12} \mu+b_{12}=0$, where $a_{12}$ and $b_{12}$ are independent of $\lambda$ and $\mu$, and

$$
a_{12}=\int_{0}^{2 \pi} \phi\left((2 \kappa(x . n)-1)\left(n_{1}^{2}-n_{2}^{2}\right)-4 \kappa\left(x_{1} n_{2}-x_{2} n_{1}\right) n_{1} n_{2}\right) d s,
$$

with $x . n=x_{1} n_{1}+x_{2} n_{2}$.

REMARK 1. In [2], $a_{12}$ is not given explicitly, but it can be easily obtained from the proof (see also [3]). We do not need the precise formula for $b_{12}$.

Now using the residue formula we obtain

$$
\int_{0}^{2 \pi} \phi^{2}\left(x_{1} n_{2}-x_{2} n_{1}\right) n_{1} n_{2} d s=\frac{\pi a^{3} b^{3}\left(a^{2}-b^{2}\right)}{4\left(a^{2}+b^{2}\right)^{2}},
$$




$$
\int_{0}^{2 \pi}\left(x_{1} n_{2}-x_{2} n_{1}\right) n_{1} n_{2} d s=\frac{\pi a b(a-b)}{a+b},
$$

and

$$
a_{12}=-\frac{\pi a b(a-b)^{3}}{(a+b)\left(a^{2}+b^{2}\right)} .
$$

LEMma 2. We have

$$
\lambda \frac{a^{2} b^{2}(a+b)}{4\left(a^{2}+b^{2}\right)^{2}}+\left(\mu^{2}-1\right) \frac{1}{a+b}+(1-\mu) \frac{(a-b)^{2}}{(a+b)\left(a^{2}+b^{2}\right)}=0 .
$$

Proof. Since Lemma 1 holds for $\lambda=0$ and $\mu=1$ we get

$\lambda \int_{0}^{2 \pi} \phi^{2}\left(x_{1} n_{2}-x_{2} n_{1}\right) n_{1} n_{2} d s+\left(\mu^{2}-1\right) \int_{0}^{2 \pi}\left(x_{1} n_{2}-x_{2} n_{1}\right) n_{1} n_{2} d s+a_{12}(\mu-1)=0$, and the result follows by using (6)-(8).

Lemma 3. We have

$$
\mu=1-\lambda \frac{a^{2} b^{2}}{4\left(a^{2}+b^{2}\right)} .
$$

Proof. When $\lambda=0$ we have $u=\mu \chi$ and (5) implies that $\mu=1$. If $\lambda>0$ we define

$$
w(x)=\cosh \frac{\sqrt{\lambda} a b}{\sqrt{a^{2}-b^{2}}}\left(\frac{x_{1}}{a}+i \frac{x_{2}}{b}\right), \quad x \in \mathbb{R}^{2},
$$

and if $\lambda<0$ we define

$$
w(x)=\cos \frac{\sqrt{-\lambda} a b}{\sqrt{a^{2}-b^{2}}}\left(\frac{x_{1}}{a}+i \frac{x_{2}}{b}\right), \quad x \in \mathbb{R}^{2} .
$$

We have

$$
\Delta w+\lambda w=0 \quad \text { in } \mathbb{R}^{2} .
$$

Now using Green's formula we can write

$$
\begin{aligned}
\lambda \int_{\Omega} w u d x & =-\int_{\Omega} u \Delta w d x \\
& =-\int_{\Omega} w \Delta u d x+\int_{\partial \Omega} w \frac{\partial \chi}{\partial n} d \sigma \\
& =\lambda \int_{\Omega} w u d x+\mu \int_{\Omega} w d x+\int_{\partial \Omega} w \frac{\partial \chi}{\partial n} d \sigma,
\end{aligned}
$$

from which we deduce that

$$
\int_{\partial \Omega} w \frac{\partial \chi}{\partial n} d \sigma+\mu \int_{\Omega} w d x=0
$$


We have

$$
\int_{\Omega} w d x=\pi a b
$$

and

$$
\int_{\partial \Omega} w \frac{\partial \chi}{\partial n} d \sigma=\sum_{n \geq 0} \frac{\lambda^{n} a^{2 n} b^{2 n}}{(2 n) !\left(a^{2}-b^{2}\right)^{n}} I_{n}
$$

where

$$
I_{n}=-\frac{a b}{a^{2}+b^{2}} \int_{0}^{2 \pi}\left(a^{2} \sin ^{2} \theta+b^{2} \cos ^{2} \theta\right) e^{2 i n \theta} d \theta
$$

Since

$$
I_{0}=-\pi a b, \quad I_{1}=\frac{\pi a b\left(a^{2}-b^{2}\right)}{2\left(a^{2}+b^{2}\right)}, \quad I_{n}=0 \quad \text { for } n \geq 2,
$$

the lemma follows.

Now we can complete the proof of the theorem. Assume that $\mu \neq 1$. Then Lemma 3 implies that $\lambda \neq 0$. Using Lemmas 2 and 3 we get $\mu=1$ and we reach a contradiction. Therefore $\mu=1$. By Lemma 3 we have $\lambda=0$ and the theorem follows.

We conclude this note with the following conjecture and two remarks.

ConjeCture. Disks are the only smooth bounded simply-connected open sets for which problem (1)-(2) and (5) has more than one solution.

REMARK 2. It seems clear that there is some connection between the above inverse problem and other studies related to the Schiffer conjecture. A smooth bounded simply-connected open set $\Omega$ is said to have the Schiffer property if (for any $\lambda$ ) the overdetermined boundary value problem

$$
\begin{array}{ll}
\Delta v+\lambda v+1=0 & \text { in } \Omega, \\
v=0, \quad \frac{\partial v}{\partial n}=0 & \text { on } \partial \Omega,
\end{array}
$$

has no solution. It is well known that disks do not have the Schiffer property. Indeed, let $r>0$ be such that $J_{1}(\sqrt{r})=0$. Then the function

$$
v_{r}(x)=\frac{1}{r}\left(\frac{J_{0}(\sqrt{r}|x|)}{J_{0}(\sqrt{r})}-1\right), \quad x \in \Omega,
$$

satisfies (9)-(10) with $\lambda=r$ when $\Omega$ is the unit disk.

The Schiffer conjecture asserts that disks are the only smooth bounded simply-connected open sets for which (9)-(10) has a solution for even a single value of $\lambda$. It has been shown ([6]) that for smooth bounded simplyconnected open sets the Schiffer property is equivalent to the Pompeiu property. We shall not define the latter, instead we refer the reader to the bibliographic survey of the Pompeiu problem ([7]). 
REMARK 3. Notice that the argument used in the proof of Lemma 3 provides a very simple proof that true ellipses have the Schiffer property. Indeed, assume that (9)-(10) has a solution. Multiplying (9) by $w$ and using Green's formula and (10) we arrive at

$$
\int_{\Omega} w d x=0
$$

and we get a contradiction.

\section{References}

[1] J. Blum, Numerical Simulation and Optimal Control in Plasma Physics, Wiley and Gauthier-Villars, New York, 1989.

[2] R. Dalmasso, An inverse problem for an elliptic equation with an affine term, Math. Ann. 316 (2000), 771-792.

[3] —, An inverse problem for an elliptic equation, Publ. RIMS 40 (2004), 91-123.

[4] M. Vogelius, An inverse problem for the equation $\Delta u=-c u-d$, Ann. Inst. Fourier (Grenoble) 44 (1994), 1181-1209.

[5] G. N. Watson, A Treatise on the Theory of Bessel Functions, 2nd ed., Cambridge Univ. Press, 1962.

[6] S. A. Williams, A partial solution to the Pompeiu problem, Math. Ann. 223 (1976), 183-190.

[7] L. Zalcman, A bibliographic survey of the Pompeiu problem, in: Approximation by Solutions of Partial Differential Equations, B. Fuglede et al. (eds.), Kluwer, 1992, 185-194.

Laboratoire LMC-IMAG

Équipe EDP

Tour IRMA - BP 53

38041 Grenoble Cedex 9, France

E-mail: Robert.Dalmasso@imag.fr 